\title{
Nuevas intervenciones en la Cova Gran y la Cova Freda de Montserrat (Collbató, Barcelona) casi 100 años después*
}

\author{
New archaeological excavations at Cova Gran and Cova Freda de Montserrat (Collbató, \\ Barcelona) after almost 100 years
}

\section{F. Xavier Omsa, Juan I. Morales ${ }^{a}$, Artur Cebrià ${ }^{a}$, Josep Mestres ${ }^{b}$ y Josep M. Fullola}

\section{RESUMEN}

En este trabajo se presentan los primeros datos estratigráficos, radiocarbónicos y de cultura material de las recientes excavaciones en las Coves de Montserrat (Cova Gran y Cova Freda). Estos emblemáticos yacimientos fueron excavados el año 1922 y proporcionaron uno de los primeros conjuntos cardiales de la península ibérica. Desde entonces, no volvieron a ser intervenidos de forma oficial y quedaron abandonados. En el año 2018 se han reiniciado los trabajos con sondeos en ambas cuevas, determinando la preservación de depósitos arqueológicos estratificados en algunos de ellos. En la Cova Freda se ha reconocido la existencia de un tramo de la cavidad probablemente usado como redil durante el Neolítico Antiguo Cardial y, en la Cova Gran, se han documentado un nivel y una estructura negativa de esa misma cronología, así como un potente nivel finipaleolítico.

\begin{abstract}
This work presents the first stratigraphic, chronological and artifactual results of the recent archaeological excavations in the Coves de Montserrat (Cova Gran and Cova Freda). These emblematic sites were excavated in 1922 and provided one of the first Cardial Ware assemblages of the Iberian Peninsula. Authorized excavations were resumed in 2018 with test pits in both caves. These showed the preservation of some stratified archaeological deposits. In Cova
\end{abstract}

Freda part of the cavity probably used to stall livestock during the Early Neolithic. In Cova Gran an Early Neolithic layer and pit are preserved, as well as a layer dating to the Epipalaeolithic.

Palabras clave: Cova Gran; Cova Freda; NE de la península ibérica; Neolítico Antiguo; Epipaleolítico; Dataciones C14.

Key words: Cova Gran; Cova Freda; NE Iberia; Early Neolithic; Epipalaeolithic; Radiocarbon dates.

\section{INTRODUCCIÓN}

La Cova Gran y la Cova Freda se sitúan en la vertiente sur del macizo de Montserrat, en la localidad de Collbató (Baix Llobregat, Barcelona), a escasos 30 $\mathrm{km}$ de Barcelona. Sus coordenadas UTM ETRS89 31N son Cova Gran= X 402502, Y 4603198; Cova Freda= X 402640, Y 46030110 (Fig. 1A). Ambas están casi a la misma altitud, 500 y $502 \mathrm{~m} \mathrm{~s}$. n. m. respectivamente, abiertas en el contexto general del macizo: conglomerados con interfases de lutitas. Forma la Cova Gran una única galería de unos $35 \mathrm{~m}$ de largo E-NE con una boca de unos $7 \mathrm{~m}$ de alto por $8 \mathrm{~m}$ de ancho. Gradualmente va descendiendo hasta hacerse impenetrable. Al final de la cavidad, a la derecha, existe un

\footnotetext{
* Las intervenciones arqueológicas en la Cova Gran y la Cova Freda se integran dentro del proyecto "Transicions culturals durant el Plistocè superior i l'Holocè al litoral-prelitoral central de Catalunya" (CLT009/18/00024, 2018-2021 de la OSIC de la Generalitat de Catalunya), en el marco del grupo de investigación SERP de la Universitat de Barcelona. Sus proyectos SGR2017-00011 y HAR2017- 86509 han sufragado las dataciones de este artículo. J. I. M goza de un contrato postdoctoral Juan de la Cierva-Incorporación (IJCI-2017-31445) del Ministerio de Ciencia, Innovación y Universidades. Agradecemos al ayuntamiento de Collbató y al Patronat de la Muntanya de Montserrat su apoyo económico y en infraestructuras.

a SERP, Seminari d'Estudis i Recerques Prehistòriques. Secció de Prehistòria i Arqueologia. Universitat de Barcelona. C/ Montalegre 6-8, 08001 Barcelona. Correos e.: FXO oms@ub.edu https://orcid.org/0000-0002-1642-548X; JIM jimorales@ub.edu https://orcid.org/0000-0002-8253-414X; AC arturcebria@gmail.com https://orcid.org/0000-0002-5674-4135; JMF fullola@ub.edu https://orcid.org/0000-0001-7089-1425

b VINSEUM, Museu de les Cultures del Vi. Plaça de Jaume I 1, 08720 Vilafranca del Penedès (Barcelona). Correo-e.: josepmestres@hotmail.com https://orcid.org/0000-0002-7839-7082

Recibido 13-II-2019; aceptado 24-IV-2019.
}

Copyright: (C) 2019 CSIC. This is an open-access article distributed under the terms of the Creative Commons Attribution 4.0 International (CC BY 4.0) License 

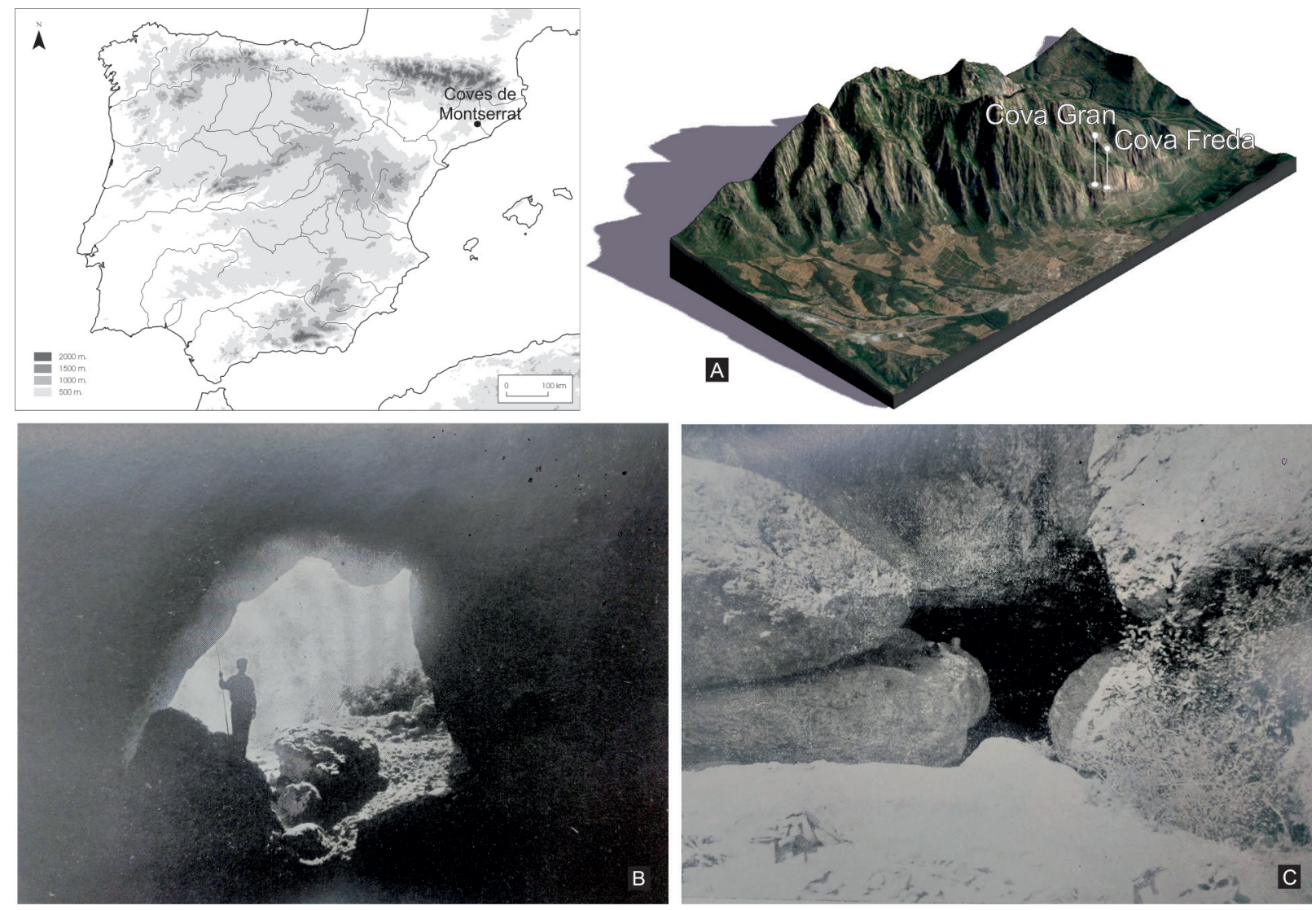

Fig. 1. A. Localización de las "Coves de Montserrat" (Collbató, Barcelona) en la península ibérica y en el macizo de Montserrat; B. vista desde el interior de la Cova Gran (Colomines 1925: lám. VIII) y vista de la boca de la Cova Freda (Colomines 1925: lám. XXVII) (autoría SERP, Universitat de Barcelona) (A. en color en la versión electrónica).

divertículo en forma de corta galería que parece vaciada recientemente. La Cova Freda tiene una estructura más compleja. La cavidad mide $232 \mathrm{~m}$ de longitud con un desnivel máximo de $16 \mathrm{~m}$ y una orientación aproximada N-S, con una pequeña apertura original que fue cerrada con una puerta hace unas décadas. Esta puerta da paso a una sala de pequeñas dimensiones (Sala I) de unos 3,5 m de largo por $12 \mathrm{~m}$ de ancho, la única que recibe parcialmente luz del exterior. Un estrecho corredor da acceso a la Sala II de creciente inclinación hacia el interior. Mide unos $10 \mathrm{~m}$ de largo por $6 \mathrm{~m}$ de ancho. Está afectada por importantes procesos gravitacionales y cuenta con diferentes espacios laterales (entre ellos una galería de unos $20 \mathrm{~m}$ de largo, en sentido N) y coladas. A través de una rampa se accede a la Sala III (en algunas ocasiones, se ha dividido esta sala en dos), de dimensiones mayores a la anterior y con una importante presencia de grandes bloques desprendidos. Uno de ellos ha provocado que algunas topografías hayan dividido esta sala en dos.
Ascendiendo por uno de los bloques se accede a la Sala IV, la última, también con una gran cantidad de bloques y formaciones estalagmíticas descalcificadas.

La Cova Gran y la Cova Freda fueron uno de los primeros conjuntos excavados en el Mediterráneo occidental que proporcionaron cerámicas cardiales (Colomines 1925). Al desconocerse, por entonces, paralelos se denominaron cerámicas "Montserratines". Sin embargo, la metodología utilizada en su excavación no permitió situarlas a nivel estratigráfico. Por ese motivo fueron datadas al final de la Cultura de las Cuevas, en el Eneolítico. Hasta ese momento, en el NE peninsular, solamente habían sido publicadas cerámicas similares en la Cova de Can Pascual (Castellví de la Marca) (Bosch Gimpera 1923), datadas en el mismo periodo. Poco después, el año 1936 se publicaron los resultados de la excavación de 1928 en la Esquerda de les Roques del Pany (Torrelles de Foix). Allí, Martí Grivé (1936) detectó una cierta estratigrafía: primero un estrato con cerámica "argárica" (por las carenas, 
que ahora situaríamos en la Edad del Bronce), seguido por un nivel campaniforme con inhumaciones colectivas y, en la base, un nivel con un mínimo de 12 inhumaciones asociadas a cerámica "montserratina" y numerosa industria ósea (p. ej. 4 cucharas entre enteras y fragmentadas) y restos malacológicos. La Cova Bonica de Vallirana fue excavada en junio de 1936, justo antes del inicio de la Guerra Civil. Los resultados de esa campaña no fueron publicados. Por lo que desconocemos si Josep de Calasanç Serra Ràfols intuyó también alguna secuencia en esa cavidad (Baldellou 1974).

La publicación de las excavaciones de la Grotta de l'Arene Candide de Finale-Ligure (Italia) y de la Cova de la Sarsa de Bocairent fueron determinantes para empezar a delimitar la cronología y ámbito de la cerámica cardial. El yacimiento ligur localizaba las cerámicas impresas en un nivel subyacente a otros con cerámicas VBQ (Vasi a Bocca Quadrata), al mismo tiempo que se dotaba al concepto "Cardial" de una presencia pan-mediterránea (Martí 2008). Gradualmente, en el NE de la península ibérica, se fueron identificando nuevos enclaves con presencia de cerámica cardial, aunque las anotaciones estratigráficas continuaban siendo prácticamente nulas: Cova de Can Montmany en Pallejà (Colomines 1947), Cova del Bolet en Mediona (Giró 1947), Cova de la Font Major en l'Espluga de Francolí (Vilaseca 1969) y Roc d'en Sardinyà en Vilassar de Dalt (Baldellou 1972) entre otros. Finalmente, a inicios de los 1980, se empiezan a publicar las primeras estratigrafías con cerámica cardial en el NE peninsular. Destacan las de la Cova del Toll de Moià (Guilaine et al. 1981), la Cova del Frare de Matadepera (Martín et al. 1985) y de Les Guixeres de Vilobí de Sant Martí Sarroca (Baldellou y Mestres 1981; Mestres 1981-1982), casi al mismo tiempo que las primeras definidas para territorios vecinos, en la Cova de l'Or de Beniarrés (Martí 1977) y en la Cueva de Chaves (Baldellou y Castán 1983).

Durante buena parte del siglo XX, las "Coves de Montserrat" fueron un referente por ser uno de los primeros lugares donde se documentó una ocupación cardial y por su numerosísima colección arqueológica (especialmente cerámica). Sin embargo, gradualmente fueron perdiendo peso en las publicaciones por su falta de estratigrafía conocida y de información útil desde una perspectiva actual. A inicios de la década de los 1980, Josep Mestres, Josep Tarrús y Ramon Ten analizaron y dibujaron todo el material neolítico de Cova Gran y Cova Freda, aunque esos resultados quedaron prácticamente inéditos (Mestres et al. 1983a, Mestres et al. 1983b; Mestres 1989). Recientemente, una parte han sido incluidos en tesis doctorales sobre industria lítica (Palomo 2012) y ce- rámica (Oms 2014) y en un trabajo de final de máster inédito ${ }^{1}$.

\section{LAS EXCAVACIONES DE 1922}

Un acuerdo entre los monjes de la Abadía de Montserrat y los miembros de la Secció HistòricoArqueològica del Institut d'Estudis Catalans (a partir de ahora IEC) organizó la "exploración" de las cuevas ya conocidas de la vertiente sur de la montaña, cerca de Collbató. Dirigió la intervención Josep Colomines, que había ingresado en el IEC, como técnico conservador, el año 1915. Los trabajos, discontinuos, se hicieron entre abril y octubre de 1922. El año 1925 se publicó la memoria de las excavaciones (Colomines 1925) en Cova Freda y Cova Gran y de otras más modestas: Cova del Salnitre, Coves de Santa Cecília y el sepulcro de fosa de El Bruc.

La información sobre las excavaciones y la documentación gráfica era realmente escasa (Fig. 1B y 1C) en la citada monografía, dedicada en su mayor al análisis de los materiales. En referencia a Cova Gran, se afirma que buena parte de ellos apareció en una única capa superficial de unos $25 \mathrm{~cm}$ de potencia de sedimento oscuro y carbonoso y, al mismo tiempo, que parte del conjunto afloraba al voltear los grandes bloques existentes en la mayor parte de la cueva. Sobre la Cova Freda se proporcionaron más detalles: en la Sala I se documentaron sedimentos revueltos y muy pobres, con un grosor máximo de $40 \mathrm{~cm}$. La Sala II era, según Colomines, la más intacta al conservar en algunos puntos una potencia de $2,5 \mathrm{~m}$. Detalla que un primer nivel estaba bastante revuelto, aunque se documentaron dos inhumaciones (boca arriba) cubiertas de cerámica ibérica. En un nivel inferior, con un sedimento más oscuro y de aspecto grumoso, apareció mucho material revuelto de diferentes fases. En la Sala III, bajo los bloques, continuaba el segundo nivel de la sala anterior, esta vez con una potencia de 1,5 m. En la Sala IV (en realidad una extensión de la Sala III) sólo documentó materiales en una covacha ya que el resto de la sala no contenía sedimentos. En la Sala V (de hecho, la IV) solamente se citan cerámicas informes en superficie.

\section{LAS NUEVAS CAMPAÑAS}

El mes de junio de 2018 se llevaron a cabo las primeras excavaciones reguladas en las Coves de

\footnotetext{
1 Hernández, C. Propuesta de valorización de la colección arqueologica de la Cova Freda y la Cova Gran de Montserrat (Collbató-Baix Llobregat). Trabajo de final de máster inédito, Universitat Autònoma de Barcelona. Barcelona, 66 pp.
} 
Montserrat desde la intervención del IEC del año 1922. Las campañas consistieron en 9 días en Cova Freda (Fig. 2) y otros 9 en Cova Gran (Fig. 3), bajo la dirección de los firmantes de este trabajo. Su objetivo era comprobar el estado de conservación de ambos conjuntos, puesto que las cuevas han sido frecuentadas por excursionistas y aficionados. Por ello, presentan un deficiente estado de conservación y ha sido necesario retirar una gran cantidad de sedimentos revueltos con restos prehistóricos y subactuales.

La nomenclatura utilizada para denominar los niveles en ambos yacimientos fue diferente. En Cova Freda se utilizó la numeración romana con letras para los subniveles. En Cova Gran se recurrió a un sistema de unidades estratigráficas para determinar los paquetes sedimentarios holocenos distribuidos de forma dis- continua, siendo UE100 el removido general y, a partir de UE110, los restos de estratigrafía y estructuras neolíticas. Cuando la sedimentación tenía aspecto pleistoceno, se pasó a un criterio de unidades litoestratigráficas (siendo el nivel 200 la primera).

\subsection{Cova Freda}

Dadas las aparentemente malas condiciones de conservación de este yacimiento se realizaron sondeos de pequeñas dimensiones para comprobar la integridad estratigráfica de los diferentes sectores de la cavidad. Los trabajos arqueológicos se han centrado en las Salas I y II (Fig. 2A). En la primera sala se han realizado dos sondeos de $1 \mathrm{~m}^{2}$ que han resultado fértiles,
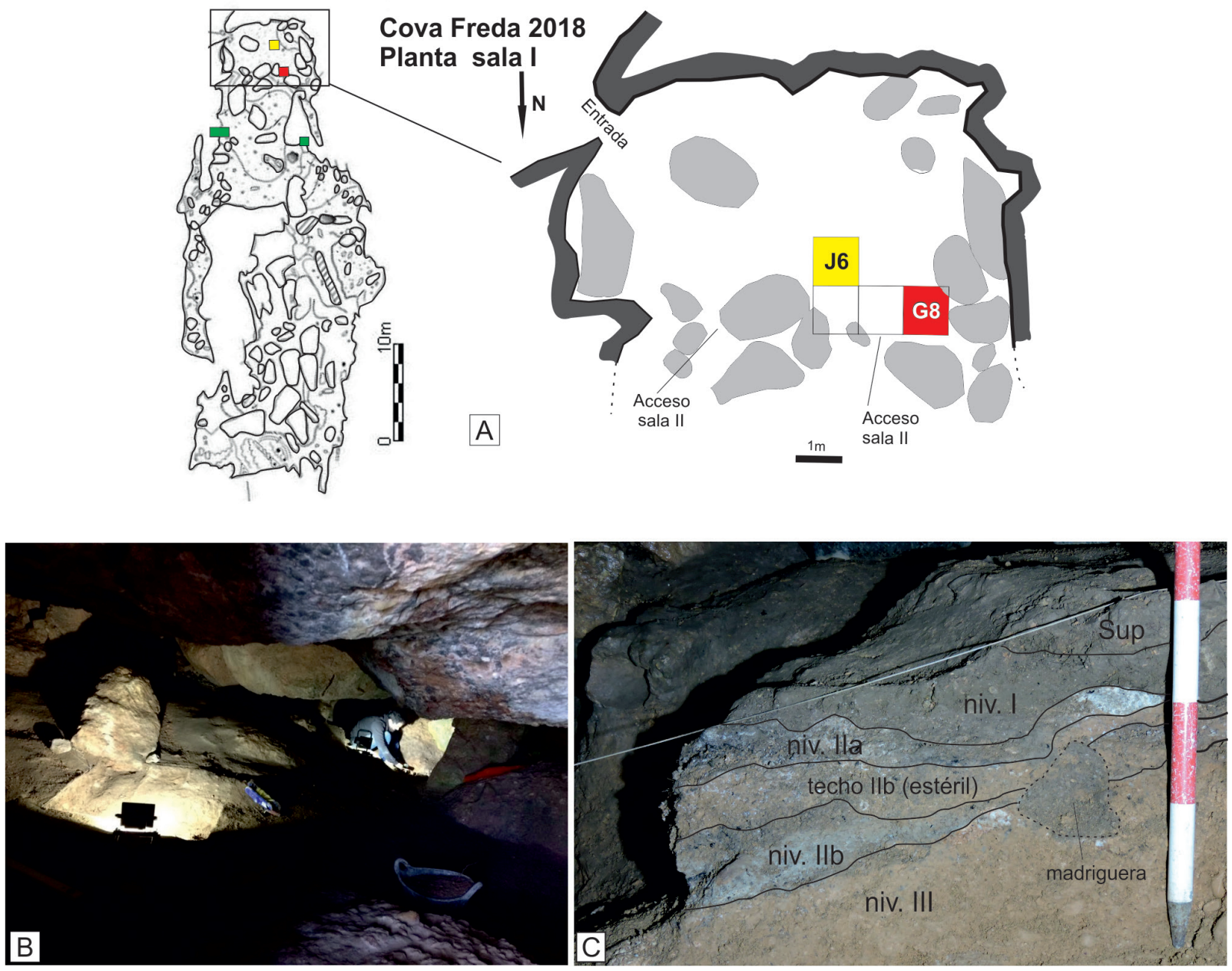

Fig. 2. Cova Freda (Collbató, Barcelona): A. planta general de la cavidad y de detalle de la Sala I, donde se localizan los diferentes cuadros excavados; B. vista de los trabajos en la Sala I; C. vista parcial de la estratigrafía (sección Este) del sondeo en el cuadro G8 (autoría SERP, Universitat de Barcelona) (en color en la versión electrónica).

Trab. Prehist., 76, N.. 2, julio-diciembre 2019, pp. 335-344, ISSN: 0082-5638

https://doi.org/10.3989/tp.2019.12241 
mientras que los dos sondeos de $2 \mathrm{~m}^{2}$ realizados en la Sala II carecían de estratigrafía pero contaban con numerosos restos arqueológicos. En la Sala I, el sondeo del cuadro J6 tiene una potencia total de $45 \mathrm{~cm}$, estando la mayoría de los estratos revueltos. Sin embargo, en la base, en un pequeño tramo (lado SE del cuadro) con un sedimento oscuro y en parte arcilloso, aparecieron materiales cerámicos informes en posición primaria. En el futuro, la ampliación de este sondeo permitirá caracterizar de manera más completa el tipo de ocupación y su cronología, así como los niveles más antiguos. El sondeo realizado en el cuadro G8 de esta sala (Fig. 2B) proporcionó interesantes resultados a nivel estratigráfico y funcional. Después de un nivel revuelto superficial (Sup), se documentó un nivel (I) in situ de coloración oscura, parcialmente arcilloso de $5-15 \mathrm{~cm}$ de potencia con numerosos restos faunísticos y cerámicos. Entre ellos había tres fragmentos peinados de tradición postcardial Molinot (Fig. 4: G8-I-4, 22,
23). Por debajo de este nivel se sucedían otros dos (IIa y IIb), separados por uno estéril (techo IIb), compuestos por laminaciones de cenizas y carbones que se extendían con un desarrollo lateral desigual por todo el cuadro. La potencia total de los dos niveles oscilaba entre los 10 y los $18 \mathrm{~cm}$ (Fig. 2C).

A la espera de los resultados de las muestras de micromorfología, su aspecto, composición y textura nos sugiere su interpretación preliminar como dos episodios diferenciados de estabulación de rebaños. Estos paquetes destacan por la abundancia de restos faunísticos. En cambio, la cerámica es escasa, informe y muy fragmentada. El nivel basal, IIb, se apoya sobre una capa de arcillas plásticas naranjas (nivel III), de la que se excavaron unos $10 \mathrm{~cm}$ de potencia, qué resultaron estériles. Todo el sedimento de los niveles IIa y IIb fue recuperado y flotado. Los numerosos restos carpológicos obtenidos están actualmente en estudio.

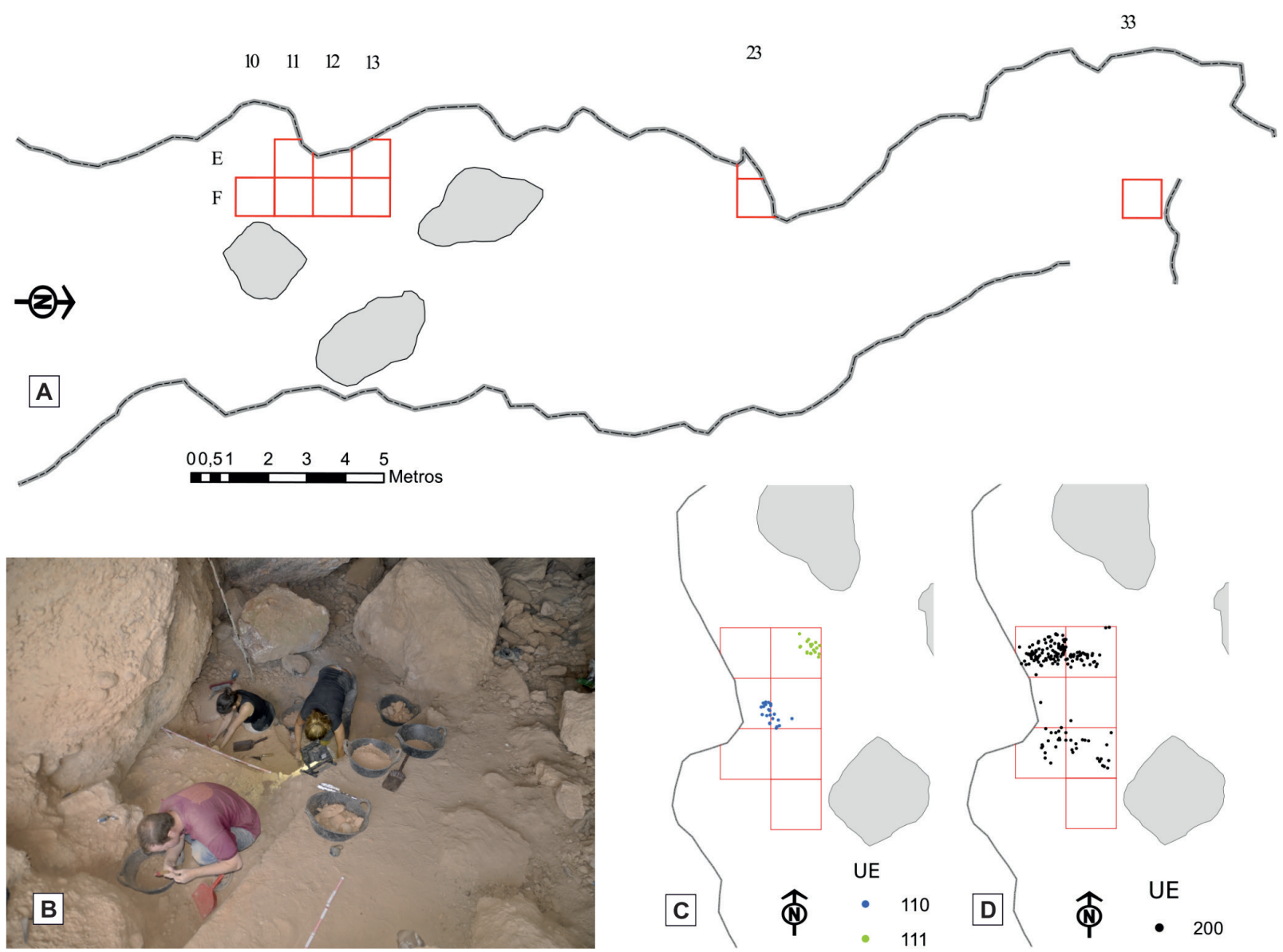

Fig. 3. Cova Gran (Collbató, Barcelona): A. planta general de la cavidad con los sectores intervenidos en 2018: B. vista de los trabajos en los cuadros E-F13 y F11 en nivel UE200; C. distribución del material de los niveles UE110 y UE111; D. distribución del material del nivel UE200 (autoría SERP, Universitat de Barcelona) (en color en la versión electrónica). 


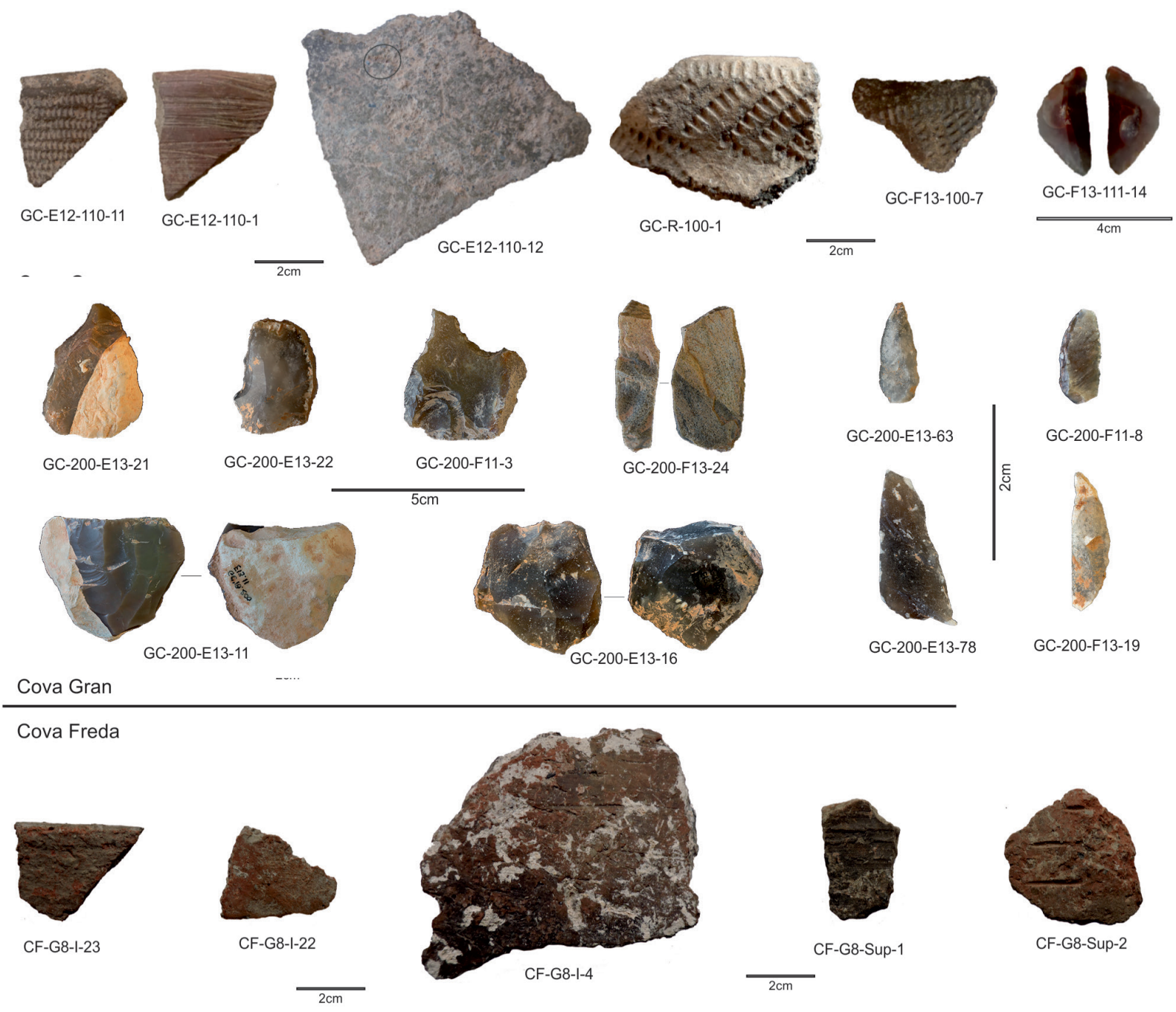

Fig. 4. "Coves de Montserrat" (Collbató, Barcelona): cerámica e industria lítica de la Cova Gran (tramo superior) y cerámica de la Cova Freda (tramo inferior). Campaña de 2018 (autoría SERP, Universitat de Barcelona) (en color en la versión electrónica).

Los sondeos de la Sala II, sin secuencia arqueológica documentada, constataron la importante alteración del espacio por todo tipo de actuaciones furtivas. Los dos sondeos proporcionaron una amplia colección de materiales situables entre el Neolítico antiguo y la época ibérica, así como numerosos restos de fauna, restos humanos, etc.

\subsection{Cova Gran}

Los tres sondeos (dos de $\left.1 \mathrm{~m}^{2}\right)$ se localizaron en los tramos inicial (E-F/10-13), medio (F23) y final (F33) de la cavidad, donde no había grandes bloques.
En los que se realizaron más al interior, los niveles están revueltos. En ellos se han recuperado numerosos restos arqueológicos, entre los que destaca la cerámica cardial, la industria lítica, principalmente fragmentos de láminas y numerosos restos humanos de pequeño tamaño que no habían sido documentados en las excavaciones de 1922. El tercer sondeo, situado en el vestíbulo, se inició con $4 \mathrm{~m}^{2}$ en forma de trinchera longitudinal ampliándose hasta los $7 \mathrm{~m}^{2}$ a medida que la aparición de grandes bloques de conglomerado exigía ampliar la superficie. En este sondeo, tras retirar una gran cantidad de sedimentos revueltos, se detectó una amplia estratigrafía que no ha sido agotada. En primer lugar, un nivel (UE100), revuelto, 
con espesor variable dependiendo del tramo, desde los casi $10 \mathrm{~cm}$ en los cuadros 11 y 12 a los casi 80 $\mathrm{cm}$ en los cuadros 14. En este último caso, asociamos la potencia con la actuación de un grupo espeleológico que realizó en esta zona un sondeo que dejó indicado en la publicación de la planimetría del yacimiento (www.espeleoindex.com). Restos de otro nivel se adosaban a la pared de la cueva. Estaba recortado por las antiguas intervenciones y protegido parcialmente por un bloque de conglomerado (UE110) (Fig. 3C). $\mathrm{Su}$ matriz estaba compuesta por cenizas y gravas y un componente limitado de arcillas de una coloración grisácea. Tenía una potencia cercana a los $30 \mathrm{~cm} \mathrm{y}$ una extensión menor a medio metro cuadrado. Este nivel contenía escasos restos, pero significativos (Fig. 4: E12-110-1,11,12): además de fauna (principalmente restos de ovicáprido), se hallaron un borde y un fragmento informe, ambos decorados mediante impresiones cardiales así como otro borde con un acabado raspado muy profundo. Esta decoración o acabado, aunque poco habitual, ya había sido detectado en los materiales antiguos (Mestres et al. 1983b: 41). En tercer lugar, a la altura de la cata realizada por el grupo espeleológico en el cuadro E13, se documentó una estructura negativa (UE111) (Fig. 3C) en parte destruida de la que se puedo excavar la base de unos $7 \mathrm{~cm}$ de potencia. Tenía una matriz oscura formada principalmente por cenizas y microcarbones. La sección de la otra mitad de la estructura (todavía no excavada de unos 30-35 cm de potencia) se conserva en buenas condiciones en el cuadro G13. En el interior de esta estructura, además de fauna y cerámica informe de igual aspecto, acabados y pastas que las de la UE110, apareció como elemento más diagnóstico un segmento de círculo con retoque a doble bisel (Fig. 3 4: F13-111-14). Todo el sedimento de las UE110 y UE111 fue recogido y posteriormente flotado, proporcionando numerosos restos carpológicos, sobre todo la UE111, actualmente en estudio.

Por debajo tanto de la UE110 como de la UE111 se documentó de forma continua en toda la extensión del sondeo la presencia de un nivel rojizo formado a partir de la disgregación de los conglomerados de la cueva (nivel 200) (Fig. 4B y 4D), formado por arenas y cantos centimétricos y decimétricos. En la zona más cercana a la boca de la cueva (cuadros F10, E-F11) aparece directamente bajo una pequeña capa de sedimento superficial de unos $10-15 \mathrm{~cm}$ de espesor. A medida que fue seguido hacia el norte, la potencia del nivel superficial aumentaba significativamente, así como la pendiente en este mismo sentido de todo el paquete. Pese a la extensión abierta, la mayor parte de la superficie presenta grandes bloques de conglomerado que han limitado en esta primera campaña la superficie excavable del nivel 200. De cara a tener una primera aproximación a su potencial, la excavación se limitó casi en exclusiva a una pequeña banqueta de unos $40 \mathrm{~cm}$ de superficie en los cuadros E-F13, en la que se excavaron unos $30 \mathrm{~cm}$ de potencia y que corresponde al corte del sondeo realizado por los espeleólogos.

El material recuperado del nivel 200 es relativamente abundante, cerca de 200 coordenados, destacando la industria lítica, los restos de fauna y los carbones. En el conjunto lítico se constata la talla microlaminar, tanto por la presencia de núcleos (Fig. 4: 200-E13$11,16)$ como de laminitas, retocadas y sin retocar, y restos de talla relacionados con el mantenimiento de los frentes de explotación. Entre el material retocado señalamos las muescas y denticulados (Fig. 4: 200E13-21,22; F11-3; F13-24), por un lado, y varias laminitas de dorso con retoque abrupto de módulo muy pequeño (Fig. 4: 200-E13-63,78; F11-8; F13-19), una en forma de segmento de círculo alargado. Una identificación preliminar de la fauna recuperada muestra restos de lepóridos con patrones de fracturación antrópica, así como de cérvidos y bóvidos pequeños, posiblemente cabra.

\section{Las dataciones}

De esta primera campaña de 2018 hemos obtenido 5 dataciones radiocarbónicas ${ }^{2}$, cuatro de Cova Gran y una de Cova Freda (Tab. 1). En Cova Gran ha sido posible datar todos los niveles excavados y en la segunda hemos priorizado fechar el nivel IIb, el más antiguo del cuadro G8, ante la falta de materiales diagnósticos. El resto de niveles de Cova Freda se datarán conforme avancen las excavaciones y se profundice en sus dinámicas de formación y su representatividad cultural.

De la Cova Gran se han realizado cuatro dataciones: una de la UE110 sobre una semilla carbonizada de Triticum c/f dicoccum $(0,01 \mathrm{~g})$ recuperada de la flotación de sedimento entre cotas de -340-355; una de la UE111 sobre una semilla carbonizada de Triticum aestivum/durum (0,013 g), recuperada de la flotación de sedimento entre cotas de -410-415; y dos del nivel 200, sobre carbón de Juniperus sp. coordenados individualmente a cotas -410 y -411 . De la UE110 se obtuvo un resultado de $6360 \pm 30$ BP (Beta-508798), 5467-5234 cal BC. De la UE111 una datación de $6380 \pm 30$ BP (Beta-508799), 5468-5310 cal BC. Mientras que las dos fechas del nivel 200 proporcionaron fechas muy similares pertenecientes a los últi-

\footnotetext{
2 Según las convenciones habituales, las calibraciones de dataciones neolíticas se presentan mediante "cal BC", y las paleolíticas mediante "cal BP". Los datos completos se encuentran en la tabla 1.
} 


\begin{tabular}{|c|c|c|c|c|c|c|c|c|}
\hline Yacimiento & Nivel & Muestra & Referencia & D13 & BP & SD & Cal BC 2o & Cal BP 2o \\
\hline Cova Gran & 110 & Triticum $\mathrm{c} / \mathrm{f}$ dicocum & Beta-508798 & -21.9 & 6360 & 30 & $5467-5234$ & $7416-7247$ \\
\hline Cova Gran & 111 & Triticum aestivum/durum & Beta-508799 & -21.5 & 6380 & 30 & $5468-5310$ & $7417-7259$ \\
\hline Cova Gran & 200 & Carbón Juniperus sp. & Beta-505684 & -23.3 & 10230 & 40 & $10166-9825$ & $12033-11847$ \\
\hline Cova Gran & 200 & Carbón Juniperus sp. & Beta-505685 & -20.9 & 10160 & 40 & $10082-9681$ & $11942-11742$ \\
\hline Cova Freda & $\mathrm{IIb}$ & Triticum $\mathrm{cf}$. aestivum/durum & Beta-508800 & -22.6 & 6410 & 30 & $5469-5327$ & $7418-7276$ \\
\hline
\end{tabular}

Tab. 1. Dataciones radiocarbónicas de la Cova Gran y la Cova Freda de Montserrat, calibradas mediante el software OxCal v. 4.3.2, con la curva IntCal'13 (Reimer et al. 2013). Los restos han sido identificados por Ethel Allué (carbones, IPHES, Tarragona) y por Ferran Antolín (semillas, UBasel, Suiza).

mos momentos del Pleistoceno, $10160 \pm 40 \mathrm{y}$ $10230 \pm 40$ BP (Beta-505684 y Beta-505685), $11842 \pm 100$ y $11940 \pm 93$ cal BP. Por último, de la Cova Freda se dató el nivel IIb a partir de una semilla carbonizada de Triticum cf. aestivum/durum (0,01 g) recuperada de la flotación de sedimento entre cotas de $-220-225$. Proporcionó una fecha de $6410 \pm 30 \mathrm{BP}$ (Beta-508800), 5469-5327 cal BC.

Las dos fechas sobre carbón del nivel 200 de Cova Gran sitúan las ocupaciones documentadas dentro del Paleolítico superior final. $\mathrm{Su}$ total coherencia interna está confirmada por los test estadísticos $\mathrm{T}$ y $\chi^{2}$ (Test $\mathrm{T}=1.5315, \chi^{2}=3.84$ a 1 grado de libertad), simplificando sus valores a una datación de $10195 \pm 28 \mathrm{BP}$, 12060-11740 cal BP. Las dataciones neolíticas de Cova Gran también son homogéneas entre sí y pertenecen a una misma fase comprendida entre $6370 \pm 21 \mathrm{BP}$, 5454-5307 cal BC (Test $\mathrm{T}=0.222, \chi^{2}=3.84$ a 1 grado de libertad). A su vez, la datación del nivel IIb de Cova Freda es coherente con las dos anteriores (Test $\mathrm{T}=1.407407, \chi^{2}=5.99$ a 2 grados de libertad), mostrando un episodio común del Neolítico Cardial en ambas cuevas entre 5465-5316 cal BC (6383 $\pm 17 \mathrm{BP})$. Estas tres dataciones se insertan perfectamente dentro del Neolítico Antiguo Cardial, en concreto en la primera mitad -Fase 1- de ese periodo según las últimas síntesis (Oms 2017).

\section{DISCUSIÓN Y CONCLUSIONES}

La primera campaña en Cova Gran y Cova Freda ha permitido una primera aproximación a su funcionalidad a pesar de la escasa extensión excavada. Las ocupaciones del Paleolítico superior final de Cova Gran supone la identificación por primera vez de secuencia paleolítica en las cuevas montserratinas. Además, son uno de los escasos ejemplos documentados en el tramo prelitoral central del NE de la península ibérica, junto con la capa 21 de la Cova de Can Sadurní y el nivel III de la Cova de la Guineu (Fullola et al. 2011; Morales et al. 2013). A la espera de que el avance de las excavaciones proporcione conjuntos más significativos que permitan profundizar en el carácter de las ocupaciones y los procesos de formación, es interesante constatar que el nivel 200 se sitúa cronológicamente en los momentos finales del GS-1 o Dryas reciente. Esta fase cuenta con numerosos datos radiocarbónicos en el NE peninsular, sin embargo, los contextos no están siempre bien definidos (Aura et al. 2011). La fase del Neolítico antiguo en Cova Gran ha sido, por el momento, definida a través de un pequeño tramo de un nivel relicto y una estructura negativa de tipo fosa-silo. Las condiciones favorables de habitabilidad de esta cueva sugieren su uso como un asentamiento puntual (del que ahora mismo no es posible determinar la intensidad) y donde, además, se llevaron a cabo prácticas de almacenaje. Por otro lado, la sala I de la Cova Freda parece responder a un lugar de estabulación de rebaños durante el Neolítico Cardial, una función secundaria lógica dadas sus escasas condiciones de habitabilidad (alta humedad, baja temperatura y la propia morfología de la cueva). Otras opciones que deberemos explorar en el futuro son la función sepulcral multifase y la obtención de agua (de sus incontables coladas) entre otras.

Las dos cuevas de Montserrat, durante el Neolítico Cardial, se integran en el conjunto de yacimientos definido como "Grupo del Llobregat" (Bernabéu 1999), recientemente reinterpretado como un complejo entramado de zonas con idiosincrasias propias y diferenciadas en cada uno de los territorios colindantes (Vallès, Penedès, Garraf-Ordal y Pla de Barcelona) (Oms 2017). Es una de las zonas donde más se ha intervenido en los últimos años y muestra un complejo mosaico de ocupación. Se conocen poblados principales al aire libre como Sant Pau del Camp (Molist et al. 2008; Gómez y Molist 2017) y Les Guixeres (Mestres y Esteve 2016; Oms et al. 2014); cuevas con fases de ocupación, inhumación y estabulación como la Cova de Can Sadurní y la Cova Bonica (Edo et al. 2011; Oms et al. 2017; Daura et al. 2019), de hábitat esporádico como la Cova del Frare y la Cova de la Guineu (Martín 2000; Oms et al. 2016), así como numerosos

Trab. Prehist., 76, N. ${ }^{\circ}$ 2, julio-diciembre 2019, pp. 335-344, ISSN: 0082-5638

https://doi.org/10.3989/tp.2019.12241 
campos de silos, como La Serreta, Mas d'en Boixos (Mestres y Esteve 2016), Pla de la Bruguera o Can Filuà entre otros. Por tanto, las "Coves de Montserrat" vienen a sumar información sobre las primeras fases de la neolitización en una de las regiones que dispone de más datos del Mediterráneo occidental.

El proyecto iniciado durante el año 2018 en la Cova Gran y la Cova Freda se va a extender los próximos cuatro años. Ante su fuerte degradación durante los últimos 100 años, el objetivo principal era determinar la existencia y conservación de estratigrafía en ambas cuevas y la posibilidad de que las secuencias, previa extracción de potentes revueltos, pudiesen ser amplias. Otro objetivo era determinar qué niveles podían estar todavía representados y se ha confirmado la conservación de niveles y estructuras del Neolítico Antiguo Cardial, mientras que otras fases posteriores todavía no han aparecido estratificadas. Asimismo, los niveles finipaleolíticos en la Cova Gran son una auténtica novedad ya que no se tenía ninguna constancia (ni por materiales descontextualizados) de la existencia de esta fase. Las próximas campañas en las "Coves de Montserrat" van a permitir extraer un mayor volumen de datos arqueoestratigráficos que van a mejorar la caracterización funcional de ambos yacimientos, así como la documentación de nuevos horizontes culturales, tanto holocenos como pleistocenos.

\section{BIBLIOGRAFÍA}

Aura, J. E.; Jordá, J. F.; Montes, L. y Utrilla, P. 2011: "Human responses to Younger Dryas in the Ebro valley and Mediterranean watershed (Eastern Spain)". Quaternary International 242 (2): 348-359. https://doi.org/10.1016/j.quaint.2011.01.023

Baldellou, V. 1972: "El yacimiento prehistórico del Roc d'en Sardinyà (Sant Genís de Vilassar, Barcelona)". Pyrenae 8: 41-52.

Baldellou, V. 1974: "Los materiales arqueológicos de la Cova Bonica de Vallirana (Barcelona)". Ampurias 36: 1-19.

Baldellou, V. y Castán, A. 1983: "Excavaciones en La Cueva de Chaves en Bastaras (Casbas- Huesca)". Bolskan 1: 9-38.

Baldellou, V. y Mestres, J. 1981: "Les Guixeres de Vilobí, un hàbitat del neolític antic a l'aire lliure". En El Neolític a Catalunya. Taula Rodona de Montserrat (Montserrat 1980): 69-74. Montserrat. Barcelona.

Bernabéu, J. 1999: "Pots, símbols and territories: the archaeological context of Neolithisation in Mediterranean Spain". Documenta Praehistorica XXVI: 101-118.

Bosch Gimpera, P. 1923: "Les coves properes a la costa catalana". Anuari de l'Institut d'Estudis Catalans VI: 476.

Colomines, J. 1925: Prehistòria de Montserrat. Analecta Montserratensia VI. Publicacions de l'Abadia de Montserrat. Montserrat

Colomines, J. 1947: "La Cueva de Can Montmany, de Pallejà". Ampurias IX-X: 237-242.

Daura, J.; Sanz, M.; Oms, F. X.; Pedro, M.; Martínez, P.; Mendiela, S.... y Fullola, J. M. 2019: "Deciphering Neolithic activities from a Cardial burial site (Cova Bonica) on the western Mediterranean coast". Journal of Archaeological Science: Reports 23: 324-347. https://doi.org/10.1016/j.jasrep.2018.10.036

Giró, P. 1947: "Cuevas del Bolet (Mediona)". Ampurias IX-X: 261.

Edo, M.; Blasco, A. y Villalba, M. J. 2011: "Guió sintètic de la prehistòria recent de Garraf'. En A. Blasco, M. Edo y M. J. Villalba (eds.):
Jornades. La Cova de Can Sadurní i la prehistòria de Garraf. Recull de 30 anys d'investigació (Begues 2008): 13-96. Milan.

Fullola, J. M.; Garcia-Argüelles, P.; Mangado, X. y Medina, B. 2011: "Paleolític i epipaleolític al Garraf-Ordal. On érem i on som..." En A. Blasco, M. Edo y M. J. Villalba (eds.): Jornades. La Cova de Can Sadurní $i$ la prehistòria de Garraf. Recull de 30 anys d'investigació (Begues 2008): 227-244. Milan.

Gómez, A. y Molist, M. 2017: "Caserna de Sant Pau del Camp (Barcelona): noves dades pel neolític antic cardial del nord-est peninsular". Cypsela 20: 11-23.

Grivé, M. 1936: "L'Esquerda de les Roques del Pany (Penedès)". Anuari de l'Institut d'Estudis Catalans VIII: 19-33.

Guilaine, J.; Llongueras, L1.; Marcet, R.; Petit, M. A. y Vaquer, J. 1981: "La cova del Toll (Moià), Barcelona". En El Neolitic a Catalunya. Taula Rodona de Montserrat (Montserrat 1980): 113-122. Montserrat, Barcelona.

Martí, B. 1977: Cova de l'Or (Beniarrés, Alicante) 1. Serie de Trabajos Varios 51, Servicio de Investigación Prehistórica, Diputación Provincial Valencia. Valencia.

Martí, B. 2008: "Cuevas, poblados y santuarios neolíticos: una perspectiva mediterránea”. En M. S. Hernández, J. A. Soler y J. A. López Padilla (eds.): IV Congreso del Neolítico Peninsular (Alicante 2006) 1: 17-27. Alicante.

Martín, A. 2000: "Els primers pastors de Sant Llonrenç del Munt al VI mil·lenni aC". En IV Trobada d'Estudiosos de Sant Llorenç del Munt i l'Obac (1997): 197-204. Barcelona.

Martín, A.; Biosca, A. y Albareda, M. J. 1985: "Excavacions arqueològiques a la Cova del Frare (Matadepera, Vallès Occidental). Dinàmica ecològica, seqüència cultural i cronologia absoluta". Tribuna d'Arqueologia 1983-1984: 91-103.

Mestres, J. 1981-1982: “Avançament a l'estudi del jaciment de les Guixeres de Vilobí”. Pyrenae 17-18: 35-53.

Mestres, J. 1989: "Los yacimientos más importantes de Cataluña". En V. Baldellou, J. Mestres, B. Martí y J. Juan-Cabanilles (eds.): El neolítico antiguo. Los primeros agricultores y ganaderos en Aragón, Cataluña y Valencia. Diputación de Huesca. Huesca: 43-48.

Mestres, J. y Esteve, X. 2016: "Sitges, cenotafis i sepulcres. 20 anys d'intervencions arqueològiques al Penedès". En X. Esteve, C. Miró, N. Molist y G. Sabaté (eds.): Jornades d'Arqueologia del Penedès 2011: 37-76. Vilafranca del Penedès, Barcelona.

Mestres, J.; Tarrús, J. y Ten, R. 1983a: "El neolític antic a Catalunya i les coves de Montserrat". Montserrat. Butlleti del Santuari $5\left(2^{\mathrm{a}}\right.$ época): 33-34.

Mestres, J.; Tarrús, J. y Ten, R. 1983b: "El neolític antic a Catalunya i les coves de Montserrat. La ceràmica". Montserrat. Butlleti del Santuari 7 ( $2^{\mathrm{a}}$ época): 39-41.

Molist, M.; Vicente, O. y Farré, R. 2008. "El jaciment de la Caserna de Sant Pau: aproximació a la caracterització d'un assentament del Neolític antic". Quaderns d'Arqueologia i Història de la Ciutat de Barcelona (Época 2) 4: 14-24.

Morales, J. I.; Cebrià, A.; Mestres, J.; Oms, F. X. y Allué, E. 2013: “La Cova del Guineu, 12000 anys de presència humana a la capçalera del Foix". III Monografies del Foix, Diputació de Barcelona. Barcelona: $172-183$.

Oms, F. X. 2014: La neolitització del nord-est de la Península Ibèrica a partir de les datacions de $14 \mathrm{C}$ i les primeres ceràmiques impreses. Tesis doctoral, Universitat de Barcelona. Barcelona. http://hdl.handle.net/2445/69672

Oms, F. X. 2017: "Fases y territorios de la neolitización en el NE de la Península Ibérica ca. 5600-4900 cal BC". Munibe 68: 155-177.

Oms, F. X.; Daura, J.; Sanz, M.; Mendiela, S.; Pedro, M. y Rodríguez, P. 2017: "First evidence of collective human inhumation from the Cardial Neolithic (Cova Bonica, Barcelona, NE Iberian Peninsula)". Journal of Field Archaeology 42: 43-53. https://doi.org/10.1080/00934690.2016.1260407

Oms, F. X.; Esteve, X.; Mestres, J.; Martín, P. y Martins, H. 2014: "La neolitización del nordeste de la Península Ibérica: datos radiocarbónicos y culturales de los asentamientos al aire libre del Penedès". Trabajos de Prehistoria 71 (1): 43-56. https://doi.org/10.3989/tp.2014.12123 
Oms, F. X.; Mestres, J.; Cebrià, A.; Morales, J. I.; Nadal, J.; Pedro, M.... y Fullola, J. M. 2016: "La cova de la Guineu (Font-Rubí, Barcelona) i les relacions plana-muntanya al Penedès durant el neolític inicial". En Del neolític a l'edat del bronze en el Mediterrani occidental. Estudis en homenatge a Bernat Marti Oliver. Serie de Trabajos Varios 119, Servicio de Investigación Prehistórica. València: 97-107.

Palomo, A. 2012: Tecnologia de la indústria lítica $i$ de la fusta de la prehistòria recent al nord- est peninsular. Anàlisi tecnomorfològica i funcional. Tesis doctoral, Universitat Autònoma de Barcelona. Barcelona. http://hdl.handle.net/10803/96270

Reimer, P. J.; Bard, E.; Bayliss, A.; Beck, J. W.; Blackwell, P. G.; Bronk Ramsey, C.... y van der Plicht, J. 2013: "IntCal13 and Marine13 radiocarbon age calibration curves $0-50,000$ years cal BP". Radiocarbon 55 (4): 1869-1887. https://doi.org/10.2458/azu js rc.55.16947

Vilaseca, S. 1969: "La Cova de la Font Major". Trabajos de Prehistoria XXVI: $117-202$

Trab. Prehist., 76, N. ${ }^{\circ}$ 2, julio-diciembre 2019, pp. 335-344, ISSN: 0082-5638

https://doi.org/10.3989/tp.2019.12241 